Qu'est ce que la roue de Sainte Catherine ? Donnez vous votre langue au chat ?

\title{
Claude Guiguen ${ }^{1}$, Hélène Guégan ${ }^{2,3}$ et Jean Pierre Gangneux ${ }^{2,3}$
}

1- Laboratoire de parasitologie, UFR de médecine, 2 avenue du professeur Léon Bernard 35043 Rennes cédex

2- Inserm U1085-IRSET, Université Rennes 1, 35000 Rennes, France

3- Laboratoire de Parasitologie-Mycologie, Centre Hospitalier Universitaire, Rue Henri le Guillou, 35033 Rennes cedex

Une fillette âgée de 8 ans arrive en consultation pour trois lésions dans le cou apparues il y a deux mois. Il s'agit d'un enfant sans antécédent médical personnel ou familial, habituellement en bonne santé. Elle a reçu il y a 1 mois un traitement antibiotique local (érythromycine) associé, devant l'absence de réponse thérapeutique, à une corticothérapie locale pendant une semaine sans résultat. Le médecin consulté à nouveau a proposé un traitement par acyclovir local Zovirax®) pour suspicion d'herpès, sans effet.

A l'examen, il s'agit d'une enfant en bon état général, pesant $25 \mathrm{~kg}$. Sur le cou il existe trois plaques, arrondies, inflammatoires, de $2 \mathrm{~cm}$ de diamètre, légèrement surélevées en périphérie avec une petite couronne de vésicules (Figure 1). Le reste de l'examen clinique est normal.

Ses deux frères plus jeunes (4 et 5 ans) ne présentent apparemment aucune dermatose. Sa sœur âgée de 10 ans, en revanche, présente une lésion érythémateuse à bordure vésiculeuse d'extension centrifuge de la jambe droite (Figure 2). L'interrogatoire précise que la famille a recueilli un jeune chat de 6 mois il y a maintenant 3 mois.

1. Énumérez les diagnostics à évoquer de principe devant cette lésion.

2. Quel diagnostic envisagez-vous en premier lieu compte tenu des données de l'interrogatoire?

3. Quel examen permettra de faire un diagnostic de certitude ? Précisez-en les conditions de réalisation et les délais de réponse attendus.

4. Quel traitement médical prescrivez-vous à ces enfants ?

5. Quelles mesures d'accompagnement proposez-vous pour préciser l'origine de la contamination et éviter la récidive ? 
1. Ces aspects circinés se rencontrent volontiers dans les lésions cutanées du lupus érythémateux disséminé, des eczémas nummulaires, du psoriasis, de l'impétigo et des mycoses cutanées [1].

2. On retiendra une épidermatophytie circinée, due à un dermathophyte zoophile ou tellurique car les lésions sont arrondies et inflammatoire. En effet, dans cette étiologie, la lésion, débute par une zone érythémateuse, souvent prurigineuse, qui s'étale et forme un anneau bien limité et d'aspect arrondi. Le bourrelet périphérique inflammatoire de cet anneau peut être recouvert de vésicules ou de bulles d'où l'appellation "herpès circiné" aujourd'hui obsolète ou de « roue de Sainte Catherine ». La zone centrale claire d'aspect cicatriciel est souvent recouverte de squames. La confluence de plusieurs lésions crée un placard polycyclique [2].

Les deux sœurs ont vraisemblablement été contaminées par le chat recueilli. La contamination à l'homme se fait en général accidentellement par contact direct (caresses) ou indirect (poils laissés sur un fauteuil, par exemple) avec un animal de compagnie (chien, chat, cochon d'Inde...), d'élevage ou de rente (chevaux, bovins...). Ces animaux peuvent être porteurs de lésions apparentes comme les "dartres des veaux », ou être "porteurs sains » comme c'est souvent le cas chez les chats avec M. canis. Les petits rongeurs sauvages peuvent aussi véhiculer des spores jusqu'à l'environnement humain par l'intermédiaire des animaux domestiques [3].

3. Le diagnostic biologique repose sur le prélèvement de squames en périphérie de la lésion à distance de toute thérapeutique locale ou générale (au moins 15 jours). Celles-ci sont raclées à l'aide d'un grattoir de Vidal ou d'une curette de Brocq. Les produits de grattage (squames) sont recueillis dans un récipient stérile.

La deuxième étape du diagnostic biologique d'une dermatophytie est l'examen direct microscopique de l'échantillon. Il est indispensable, d'abord parce qu'il affirme la présence du champignon à l'état parasitaire au sein de la zone prélevée, ensuite parce qu'il permet de donner au médecin prescripteur un premier résultat justifiant la mise en œuvre rapide d'un traitement spécifique. Les techniques (état frais avec ou sans coloration) ainsi que les produits utilisés sont largement décrits dans des ouvrages de mycologie [4]. Le dermatophyte se présente sous la forme de filaments mycéliens plus ou moins cloisonnés (arthrosporés) quelque soit l'espèce qui sera isolée par la suite. La mise en culture des prélèvements est réalisée sur des géloses de Sabouraud additionnées de cycloheximide $\left(\right.$ Actidione $^{\circledR}$ ) qui inhibe les moisissures et incubées entre $25^{\circ} \mathrm{C}$ et $30^{\circ} \mathrm{C}$. La culture peut se 
faire en tubes ou sur boîtes. La lecture des cultures se fait, habituellement, 2 fois par semaine. Le temps de pousse est d'1 à 3 semaines. L'identification repose sur l'examen macroscopique (couleur, texture) de la colonie et sur l'examen microscopique (aspect des filaments et des conidies). Le délai de rendu d'une identification d'espèce à partir d'une culture de dermatophyte est généralement de 7 à 21 jours. Les techniques de biologie moléculaire et de spectrométrie de masse peuvent permettre un diagnostic plus rapide. La connaissance de l'espèce permet de préciser l'origine de la contamination [4].

4. Il doit être débuté après le prélèvement. Il fait appel à des antifongiques locaux comme les azolés (kétoconazole, éconazole, isoconazole, bifonazole, sulconazole, miconazole ...) ou la terbinafine ou la cyclopiroxolamine ou le tolnaftate. Ces topiques antifongiques existent en crème, lotion, gel ou poudre. En cas de lésions très étendues la voie générale peut être un recours ; dans ce cas on prescrira la griséofulvine ou la terbinafine.

5. Il est nécessaire de prévoir la prise en charge du chat par un vétérinaire : examen en lumière de Wood et traitement. Il est également préconisé un nettoyage du domicile afin d'éliminer les poils de chat (chaises, fauteuils, lits, oreillers,...) [5]. La prévention est fondée sur la maitrise de la source de contamination.

\section{Références}

[1] Chabasse D, Guiguen C. Dermatophyties : les difficultés d'interprétation et les pièges du diagnostique. Revue Francophone des laboratoires, 2019, 510: 26-35.

[2]Chabasse D, Bouchara JP. Les dermatophytes : taxonomie et épidémiologie. Feuillets de Biologie $2018 ; 343: 31-42$.

[3]Monod M, Fratti M, Mignon B, Baudraz-Rosselet F, Dermatophytes transmis par les animaux domestiques. Rev Méd Suisse $2014 ; 10: 749-53$.

[4] Anofel, Dermatophytoses. In Parasitologie et Mycologie médicales. Guide des analyses et pratiques diagnostiques. Elsevier-Masson 2017, p 409-16.

[5]Robert R, Pihet M, Conventional methods for the diagnosis of dermatophytosis.Mycopatho logia.2008; 166: 295-306. 


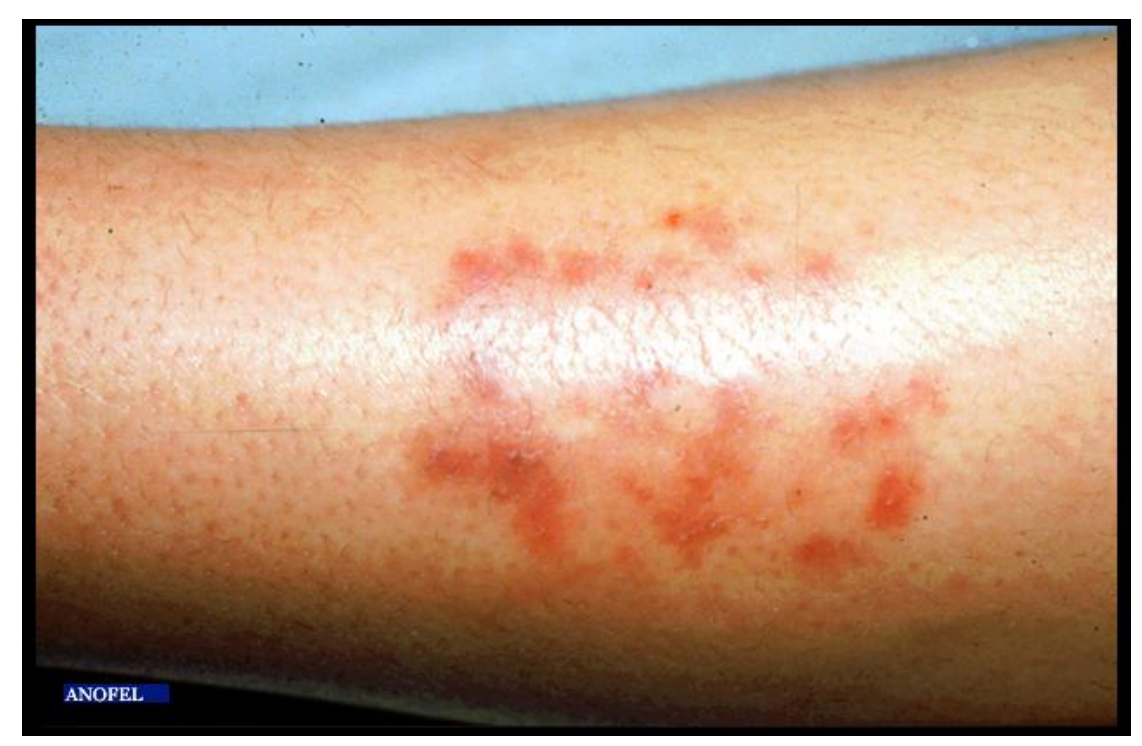

Figure 2 Lésion érythémateuse et vésiculeuses de la jambe (e.anofel)

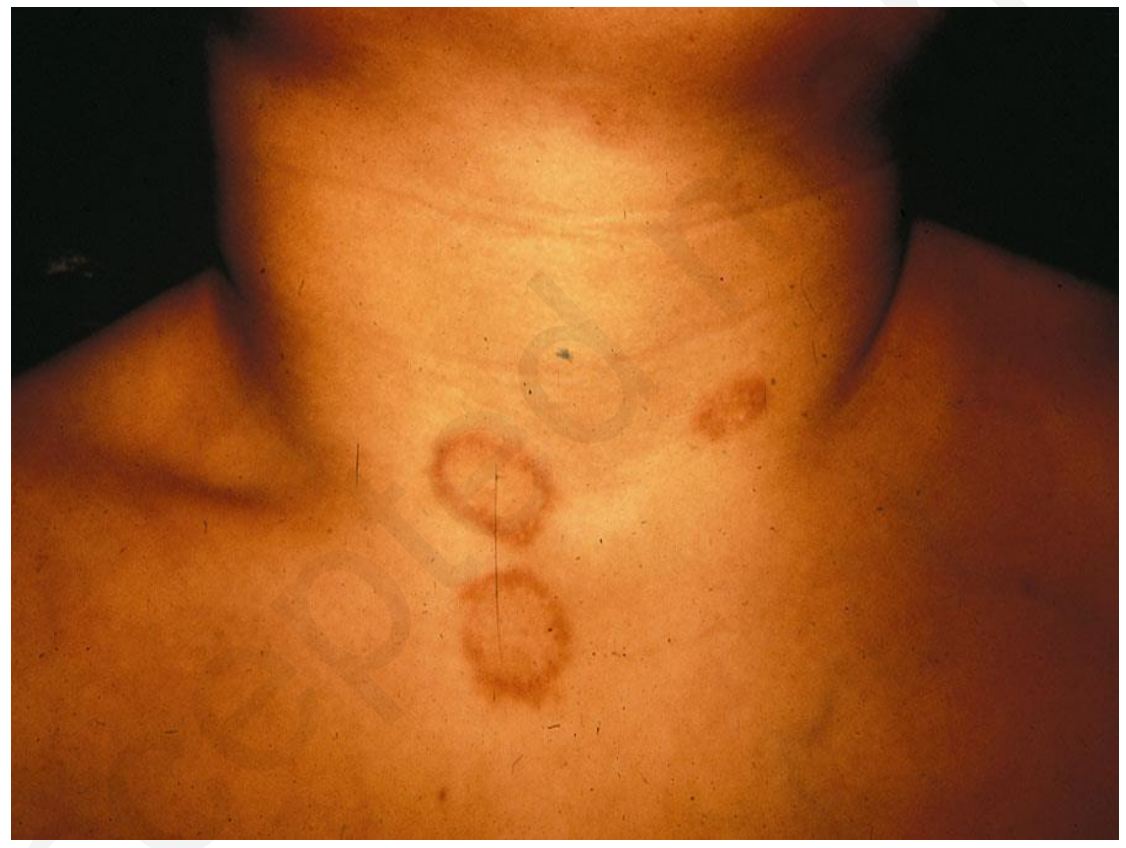

Figure 1 : Lésions circinées, vésicules et prurigineuses (Parasitologie Rennes) 